\title{
Valorisation des argiles à silex : un exemple de traitement
}

\section{Y. BOUSSAFIR}

Institut français des sciences et technologies des transports, de l'aménagement et des réseaux. 58 , boulevard Lefebvre 75732 Paris Cedex 15 yasmina.boussafir@ifsttar.fr

\begin{abstract}
Plusieurs tentatives de valorisation des silex contenus dans les argiles à silex se sont révélées peu rentables financièrement et écologiquement, car souvent très consommatrices en eau de lavage. Dans un contexte globalement de pénurie de matériau noble et sous la pression d'un contexte environnemental fort (politique européenne du zéro déchet-zéro emprunt), l'étude et la valorisation des gisements d'argile à silex redeviennent d'actualité. Le chantier de la déviation de la RN 10 entre Lisle et Pezou dans le département du Loir-et-Cher (41) a été l'occasion de montrer que l'on pouvait valoriser ces matériaux en base de structure de chaussée (couche de forme) par le biais d'un processus d'élaboration adéquat (traitement, criblage, concassage). Bien que combinant deux difficultés majeures, une forte plasticité (IP > 25) classant les sols en C1A3 au sens de la norme NF P11-300, et la présence de gros éléments (silex de taille supérieure à $200 \mathrm{~mm}$ ), il a été possible grâce aux moyens de chantier traditionnellement maîtrisés par les entreprises de terrassement en France, de valoriser le matériau. Un pré-traitement à la chaux au déblai a favorisé l'extraction des matériaux, l'abaissement de la plasticité des argiles et le désenrobage des éléments de silex de leur gangue argileuse. La reprise du stock avec criblage et concassage des éléments grossiers a permis l'homogénéisation du matériau et l'élaboration d'un $0 / 63 \mathrm{~mm}$ apte à un traitement en place au liant hydraulique.
\end{abstract}

Mots-clés : terrassements, argile à silex, traitement, concassage, criblage, couche de forme.

\section{Successfull treatment of (( argiles à silex ))}

( Argiles à silex» is the name of a geological formation from the western part of France, composed by a mix of clay material and flint. They are the result of the transformation of the chalks at the end of the Cretaceous, under specific weathering conditions. Nowadays, Flint from the "Argile à silex » formation may constitute an important source of granular material but the clay matrix avoid to used them directly like in a usual carry. Their study as cuts in the new construction of the RN 10 between Lisle and Pezou (Loir-et-Cher, 41, France) pointed the possibility to valorize the whole material after a specific process which could allow their use as the subgrade of this road. They have been tested for the realization of subgrade on the by pass of the national road RN 10 between Lisle and Pezou. This paper describes the different stages during the work area: results of studies in laboratory, lime treatments, screening, crushing and cement treatment. The results as a first experience have been successful and have really improved the process as something easy to reproduced. 


\section{Introduction}

La déviation de la RN 10 entre Lisle et Pezou remonte sur les coteaux vendômois au Nord de l'actuel tracé en traversant les formations géologiques du Tertiaire et de la fin du Secondaire. Les déblais recoupent dans leur majorité des ( argiles à silex » (Fig. 1) et des biefs à silex.1. Le mouvement des terres du tracé sur environ $6 \mathrm{~km}$ était excédentaire en matériaux.

Le premier scénario de terrassement pour la réalisation de la couche de forme était fondé sur une fourniture de matériaux en provenance d'un emprunt, constitué de graves anciennes du Loir, bien connues dans le département comme étant un matériau de très bonne qualité.

Malheureusement, le gisement étudié par le maître d'cuvre, la DDE de Loir-et-Cher, se situait au droit d'un site archéologique apparemment important : le coût des études archéologiques est vite apparu prohibitif par rapport au coût du matériau en provenance de ce site. Le maître d'œuvre s'est alors tourné vers une solution de valorisation des matériaux en provenance des déblais. Le Laboratoire régional des ponts et chaussées de Blois (LPPC Blois) a été chargé de cette étude de niveau projet.

L'étude du tracé datait de 1997. L'étude de projet de recherche d'un gisement issu des déblais pour élaborer la couche de forme a été menée en 2003. Les travaux de terrassement ont commencé deux ans plus tard avec

\footnotetext{
(1) Bief à silex : formation remaniée d'argile à silex.
}

le même maître d'œuvre et le LRPC de Blois comme contrôle extérieur des travaux de terrassement. Ceci a rendu possible une vision globale du projet. La participation du Laboratoire central des ponts et chaussée à une recherche spécifique sur cette expérimentation a permis de vérifier les caractéristiques des argiles à silex en place, après traitement au liant hydraulique.

\section{2}

\section{Caractérisation du gisement}

La déviation comportait trois déblais suffisamment importants en cubature pour constituer des gisements pour la couche de forme. Des sondages à la pelle ont été réalisés en décembre 2002 afin de reconnaitre ces trois zones (Fig. 1).

Les zones 1 et 2 ont été éliminées pour les raisons suivantes :

- l'hétérogénéité des matériaux en place avec une alternance de sables argileux, d'argiles sableuses et d'argiles, ce qui est une caractéristique des biefs à silex d'âge tertiaire. Ces hétérogénéités empêchaient de constituer un stock de caractéristiques identiques. Souvent enrichis en sable, leur plasticité est très variable ;

- la présence d'agglomérats silicifiés de silex dénommés " poudingues », qui sont également une caractéristique des altérations climatiques du Tertiaire. Ces conglomérats de taille pluri-métrique constituent une véritable gêne à l'extraction et auraient ralenti l'exploitation et la stratégie d'élaboration prévue pour la couche de forme.

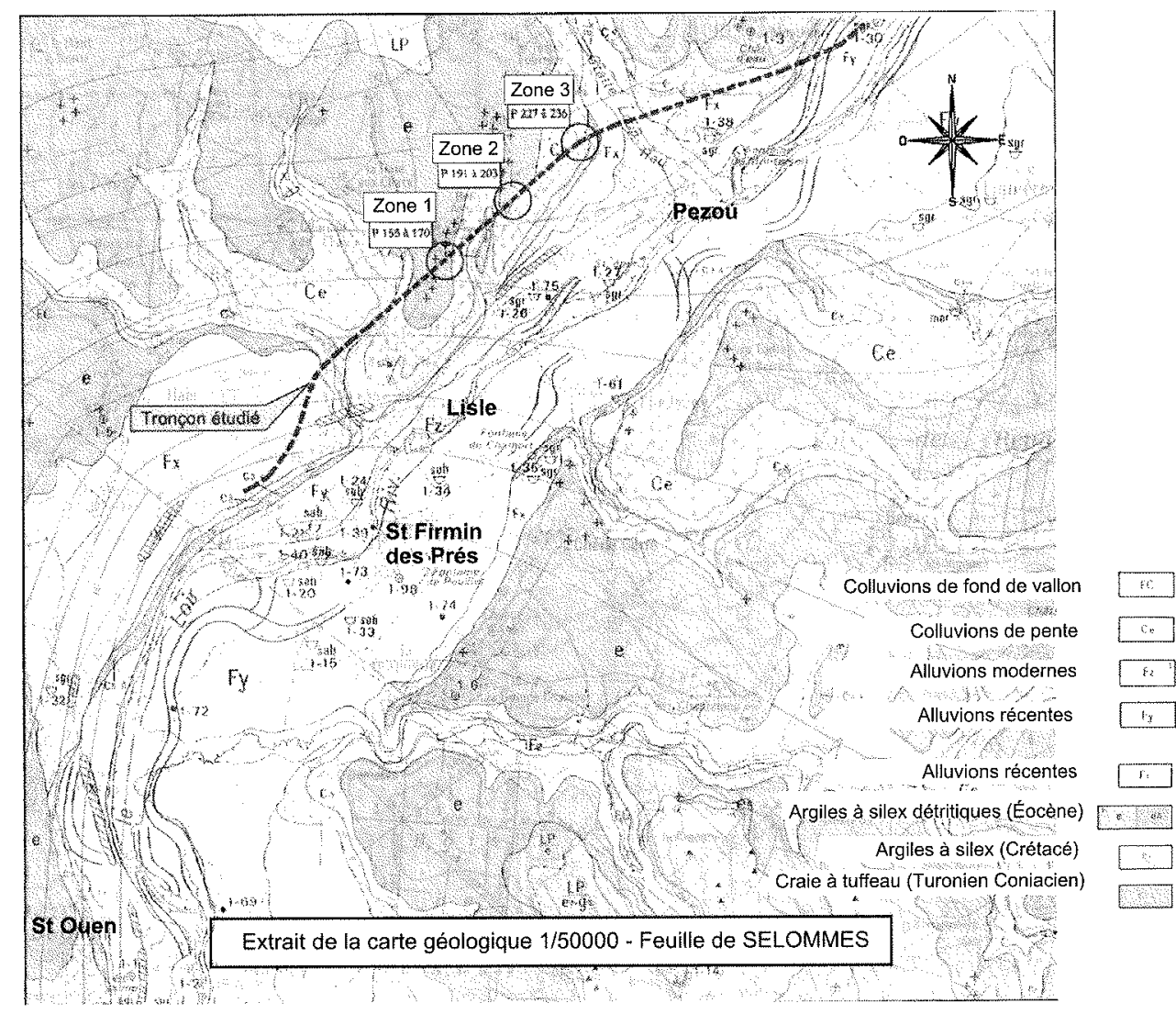

ศ6. 1 Situation géologique du projet. 
Le troisième déblai (zone 3 sur les figures 1 et 2) était le plus intéressant du projet car il présentait des volumes suffisants (environ $68000 \mathrm{~m}^{3}$ sur $300 \mathrm{~m}$ de déblai), une position judicieuse par rapport à une possible aire de stockage temporaire, et de manière relative, une meilleure homogénéité des matériaux en place. Ces matériaux correspondaient au faciès des argiles à silex de l'Éocène (autrement appelées « Formation résiduelle à silex »), très différents des formations appelées biefs à silex qui, elles, correspondent à des faciès remaniés des argiles à silex.
Les caractéristiques des matériaux rencontrés en sondages sont les suivantes:

$\mathrm{D}_{\max }=80$ à $250 \mathrm{~mm}$ (localement $300 \mathrm{~mm}$ ) ;

$<80 \mu \mathrm{m}=42,9$ à $75,2 \%$;

$<20 \mathrm{~mm}=66$ à $85 \%$;

$<50 \mathrm{~mm}=79$ à $87 \%$;

$\mathrm{IP}=24$ à 38 .

Les sols ont été majoritairement classés en $\mathrm{C}_{1} \mathrm{~A}_{3}$ au sens de la norme NFP11-300 (AFNOR, 1992).

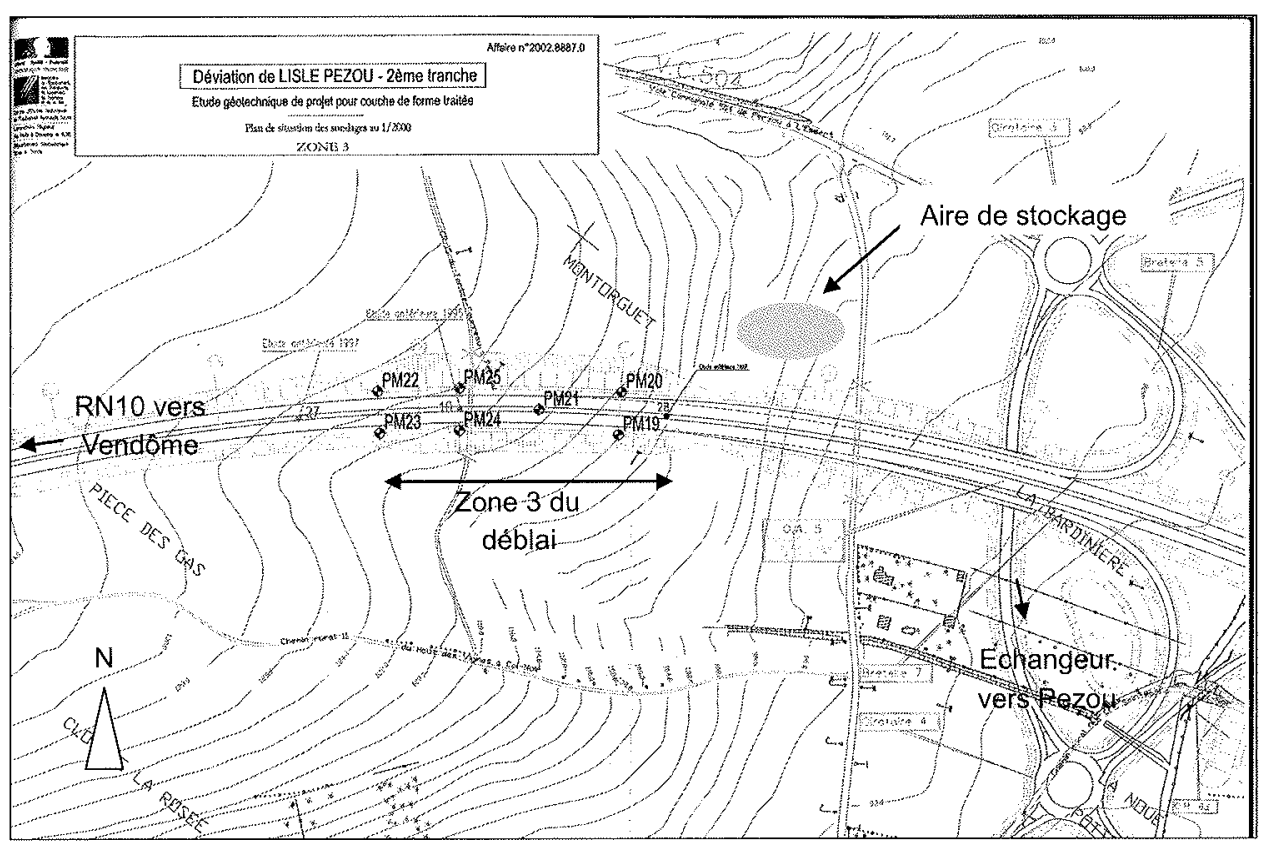

f6. 2 Localisation en plan de la zone 3 concernée; position des sondages de reconnaissance et de l'aire de stockage.

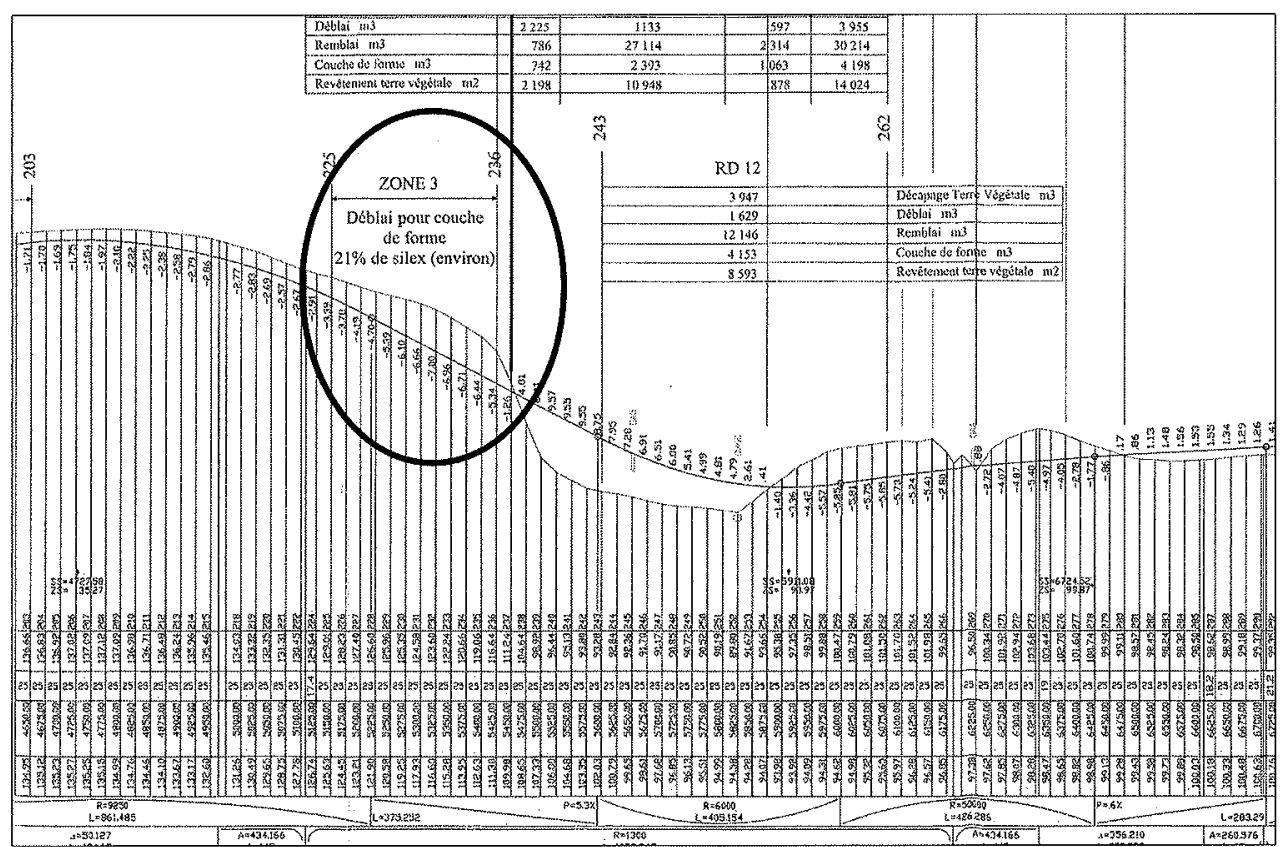

Fic. 3 Localisation de la zone 3 sur le profil en long dans l'axe du projet. 
Deux études de traitement chaux-ciment ont été programmées sur deux échantillons représentatifs du gisement. Pour le premier échantillon non traité, appelé par la suite mélange A et d'indice de plasticité = 26, les références de l'étude Proctor normal sont:

$\rho_{\mathrm{d} \text { OPN }}=1,52 \mathrm{t} / \mathrm{m}^{3}$;

$\mathrm{w}_{\mathrm{OPN}}=24,6 \%$.

Pour le deuxième échantillon non traité, appelé par la suite mélange $B$ et d'indice de plasticité $=37$, les références de l'étude Proctor normal sont :

$\rho_{\mathrm{a} \text { OPN }}=1,42 \mathrm{t} / \mathrm{m}^{3}$;

$\mathrm{w}_{\text {OPN }}=29,5 \%$.

Dans le Guide sur le traitement des sols à la chaux et/ ou aux liants hydrauliques (LCPC-SETRA, 2000), le critère d'homogénéité est basé sur la densité OPN. Dans cette étude et compte tenu de la variabilité des $\mathrm{D}_{\text {max }}$ le critère retenu pour apprécier la variabilité du gisement a été l'indice de plasticité, car cet indice semblait être l'élément le plus important pour juger de l'aptitude ou de la facilité au traitement. L'analyse de ces indices a permis de considérer le gisement comme moyennement homogène.

\section{3}

\section{Résultats de l'étude de traitement}

L'étude réalisée au titre du projet a été basée sur un pré-traitement à la chaux vive (2 et $3 \%$ ) suivi d'un traitement au ciment normalisé CEM II A/LL 32,5R, avec des dosages classiques de 4 et $6 \%$ de ciment.
Les deux mélanges ont été testés séparément. Pour cela, les matériaux ont été homogénéisés, tamisés à $20 \mathrm{~mm}$ et préparés à la teneur en eau OPN. Après malaxage dans un cutter HOBBART et incorporation du dosage en chaux et en ciment étudié, des petites éprouvettes ont été confectionnées pour étudier l'évolution des résistances en compression simple et en compression diamétrale dans le temps.

\section{1}

\section{Évolution des résistances en compression simple $\left(\mathbb{R}_{c}\right)$}

Elles ont été déterminées sur des éprouvettes compactées statiquement, de diamètre $10 \mathrm{~cm}$ et hauteur $20 \mathrm{~cm}$, moulées à 98,5\% de la densité OPN et à la teneur en eau OPN. Elles ont été écrasées après des délais de conservation de 7,28,60 et 90 jours dans des étuis étanches à $20^{\circ} \mathrm{C}$ en atmosphère saturée ou en immersion dans l'eau. Les résultats sont présentés dans le tableau I.

L'évaluation des résistances en compression simple permet de juger de l'évolution de la prise hydraulique et de sa qualité en fonction du vieillissement des éprouvettes. Les résultats ont montré qu'une prise hydraulique se développait, avec malgré tout un faible niveau de performances au bout de 90 jours. L'évolution était continue et ne semblait pas finie après 90 jours de cure, ce qui semblait un critère rassurant pour le niveau de qualité final.

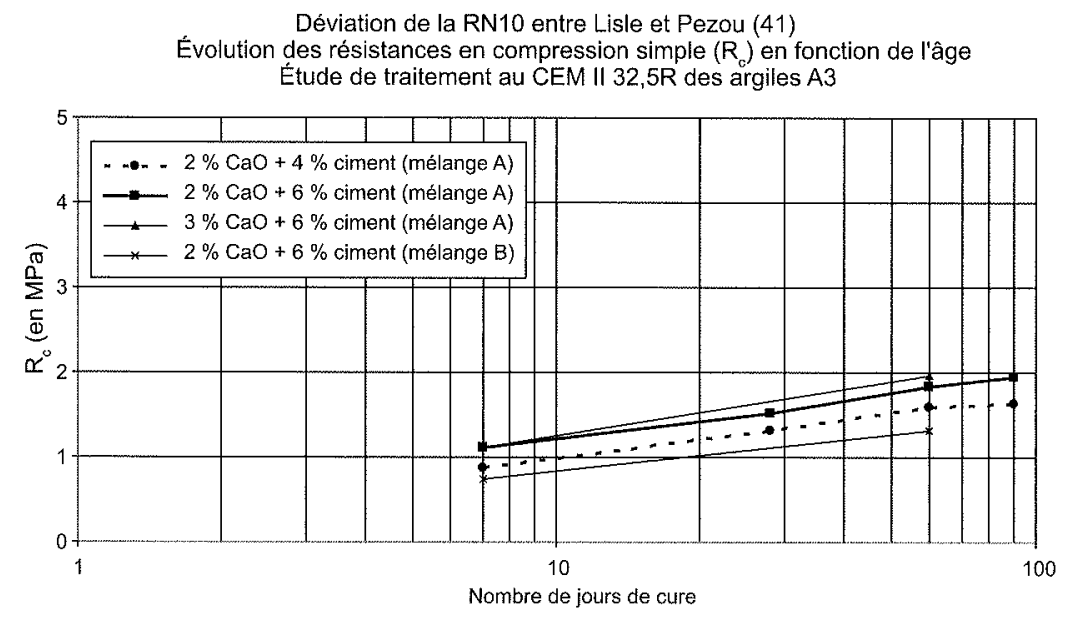

HG. 4 Évolution des résistances en compression simple des éprouvettes traitées (MPa) en fonction de l'âge (en jours de cure).

TABteuvi. Synthèse des moyennes des résistances en compression simple obtenues sur trois éprouvettes à chaque âge et à chaque dosage pour chacun des mélanges (en MPa).

\begin{tabular}{|c|c|c|c|c|c|}
\hline & & & Melange A & & Nelange 1 \\
\hline & $\%$ chaux & 2 & 2 & 3 & 2 \\
\hline & $\%$ ciment & 4 & 6 & 6 & 6 \\
\hline \multirow{5}{*}{$\begin{array}{l}\text { Âge en jours } \\
\text { de cure }\end{array}$} & 7 jours & 0,87 & 1,11 & 1,11 & 0,75 \\
\hline & 28 jours & 1,31 & 1,52 & non réalisé & non réalisé \\
\hline & 60 jours & 1,59 & 1,83 & 1,96 & 1,31 \\
\hline & 90 jours & 1,63 & 1,95 & non réalisé & non réalisé \\
\hline & $\mathrm{R}_{\mathrm{i}}^{*}$ & 0,91 & 0,95 & 0,89 & 0,58 \\
\hline
\end{tabular}

${ }^{*} R_{i}=$ rapport d'immersion à 60 jours $=R_{c}$ avant immersion $/ R_{c}$ après immersion. 


\section{Évolution des résistances en compression diamétrale $\left(R_{\mathrm{it}}\right)$}

Ces essais ont été effectués sur des éprouvettes de diamètre $10 \mathrm{~cm}$ et de hauteur $10 \mathrm{~cm}$, moulées par compactage statique à l'aide d'une presse à double effet à $96 \%$ de la densité OPN et à la teneur en eau OPN. Les moyennes des résistances en compression diamétrale $\left(R_{i t}\right.$ ou anciennement $\left.R_{t b}\right)$ et des modules $\left(E_{\mathrm{jt}}\right)$ mesurés sur les éprouvettes de sol A et B traités à la chaux et au ciment sont regroupés dans le tableau II.

Ces résultats replacés dans un diagramme $\left(R_{t}, E\right)$, classiquement utilisé pour le dimensionnement des couches de formes traitées aux liants hydrauliques, permettent de situer les performances en zones mécaniques 4 et 5 (Fig. 5).

Le classement en zone mécanique 4 ou 5 autorisant une utilisation et un dimensionnement en couche de forme traitée (Guide de traitement des Sols, LCPCSETRA, 2000), ces résultats ont permis de définir une solution pour le projet. Cette solution était basée sur un traitement en place à $2 \%$ de chaux vive suivie d'un traitement à $6 \%$ de ciment qui est la solution étudiée autorisant le meilleur classement mécanique ; le dimensionnement global de la chaussée proposé en référence au Guide de traitement des sols avec l'hypothèse d'une arase AR1 (35 MPa) permet l'obtention d'une plateforme $\mathrm{PF} 2$, avec $35 \mathrm{~cm}$ du matériau du déblai 3 traité conformément à la présente étude à $2 \% \mathrm{CaO}$ et $6 \%$ de ciment.

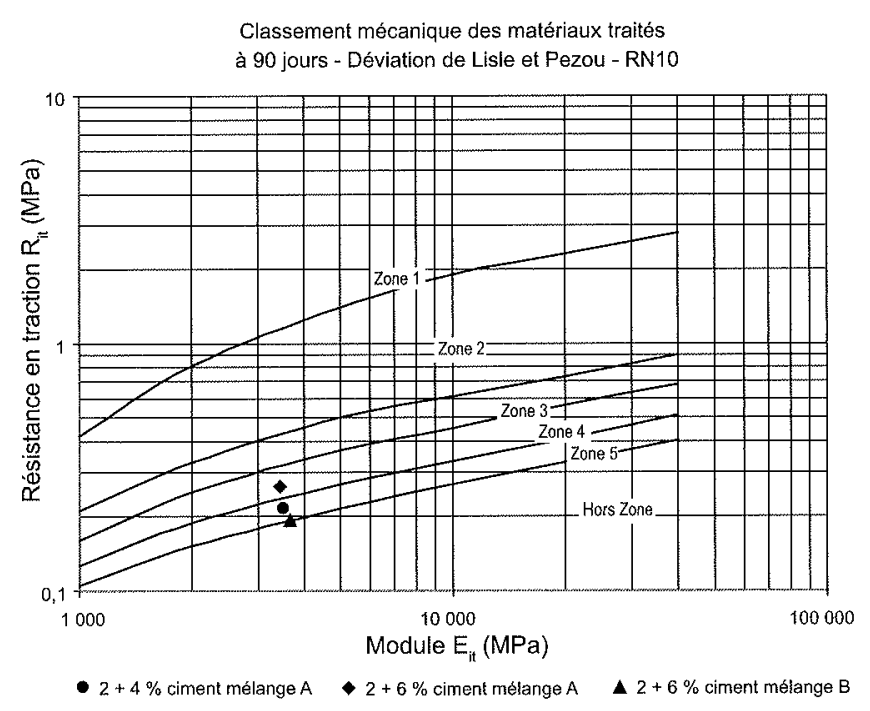

ro. 3 Résultats à 90 jours des mélanges $A$ et $B$ traités chaux et ciment reportés dans un diagramme $\left(R_{t^{\prime}}, E\right)$.
Les caractéristiques mécaniques obtenues sur les mélanges (en laboratoire) permettaient de supposer le matériau traité comme non gélif à 90 jours $\left(\mathrm{R}_{\mathrm{tb}}>0,25 \mathrm{MPa}\right.$, selon le Guide de traitement des sols $)$.

\section{4}

\section{Conduite du chantier}

\section{1)}

\section{Élaboration du matériau}

Le réemploi des argiles à silex de la zone retenue pour le projet a été entièrement défini dans le Cahier des clauses techniques particulières (CCTP) du Dossier de consultation des entreprises (DCE), appuyé par l'étude de faisabilité effectuée par le LRPC de Blois.

L'entreprise Razel retenue pour les travaux a réalisé l'ensemble des dispositions prévues avec l'application d'un Plan d'assurance de la qualité (PAQ) mis en ceuvre par son laboratoire de chantier.

La zone définie entre les profils chantiers 225 et 236 (zone 3 des figures 2 et 3 ) a fait l'objet d'un pré-traitement rustique à la chaux vive au déblai en septembre et octobre 2005 (Fig. 6). Le dosage réellement mis en œuvre, de $1,5 \%$ au lieu de $2 \%$, résulte d'une adaptation pour tenir compte des nombreux silex de taille supérieure à $50 \mathrm{~mm}$.

La méthode de traitement à la charrue a permis de traiter les matériaux quel que soit le diamètre des silex en place. Elle présentait l'avantage de fournir un niveau relativement élevé de cadence pour un taux d'usure du matériel de traitement quasiment nul. Du point de vue énergétique, il a été nécessaire de réaliser deux à trois passes de charrue pour garantir une certaine qualité d’homogénéisation entre le matériau et la chaux. La taille des « mot-

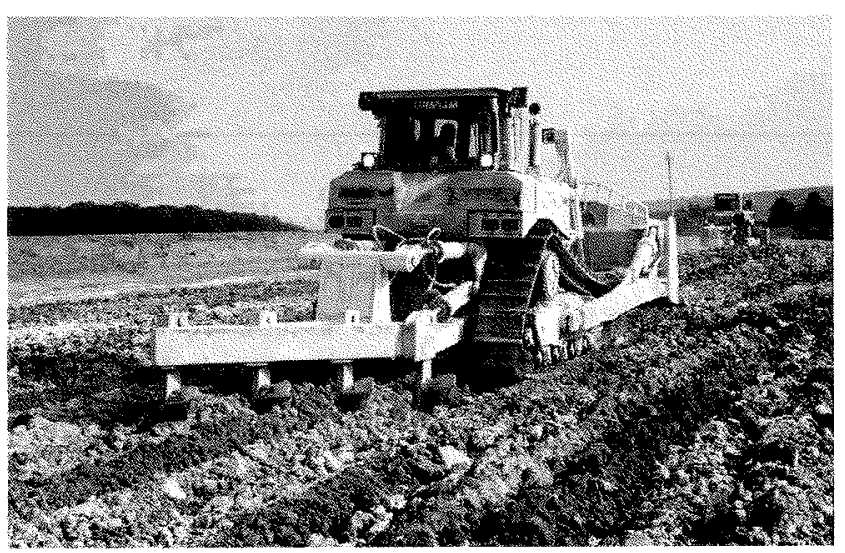

116. 6 Traitement à la chaux réalisé à la charrue à socs tractée par un bouteur.

rabruvu Synthèse des moyennes des résistances en compression diamétrale (Rit) et des modules (Eit) obtenus sur 3 éprouvettes à 90 jours pour chaque dosage et pour chacun des mélanges (en MPa).

\begin{tabular}{c|cccc}
\hline & & Melange A & Metange B \\
\hline & \% chaux incorporé & 2 & 2 & 2 \\
\% ciment incorporé & 4 & 6 & 6 \\
Écrasement & $\mathrm{R}_{\mathrm{it}}$ & 0,27 & 0,33 & 0,24 \\
à & $\mathrm{R}_{\mathrm{t}}=0,8 \times \mathrm{R}_{\mathrm{it}}$ & 0,22 & 0,26 & 0,19 \\
& $\mathrm{E}_{\mathrm{it}}$ & 3520 & 3450 & 3650 \\
\hline
\end{tabular}


tes » d'argile après traitement était d'environ 50 à $100 \mathrm{~mm}$ de diamètre. La taille des silex n'a par contre pas du tout été modifiée par ce mode de traitement et est donc restée à l'identique, soit environ 250 à 300 mm de diamètre. Une fois le matériau traité, l'extraction du mélange a été assurée par un échelon de décapeuses (motorscrapper) jusqu'à la zone de stockage temporaire située exactement au pied du déblai (localisation sur la figure 2).

Le stock a été compacté à l'aide d'un compacteur pieds dameurs de type SP2 afin de fermer le matériau et d'assurer le maintien des états hydriques jusqu'à sa reprise, prévue six mois plus tard au moment de la réalisation de la couche de forme (Figs. 7 et 8).

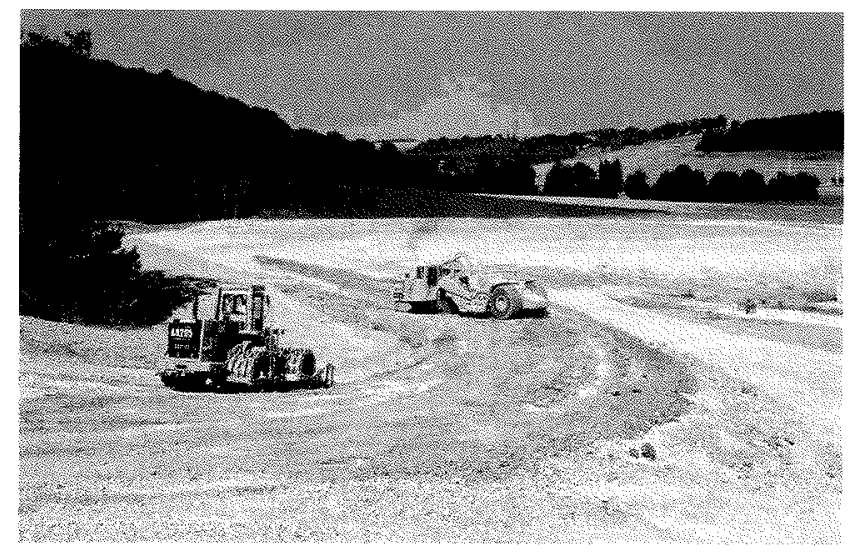

16. 7 Aire de stockage des argiles à silex traitées à la chaux vive au démarrage des travaux.

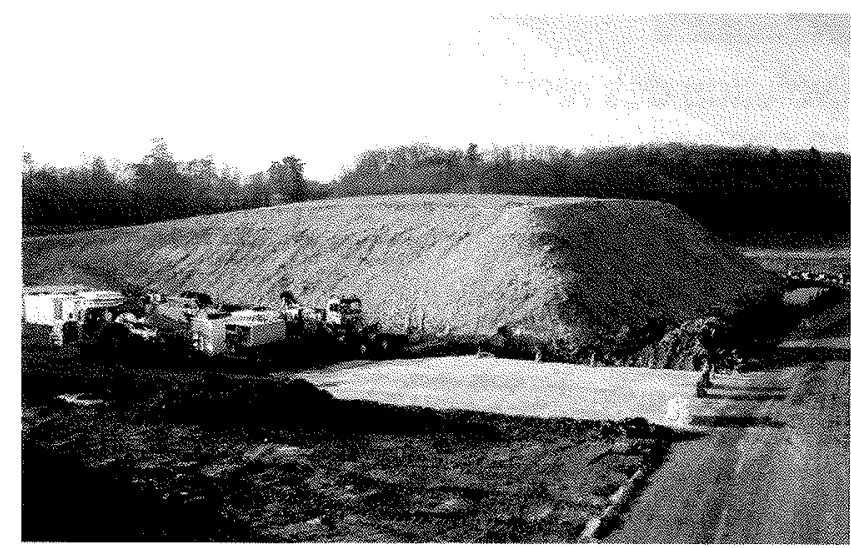

FiG 8 Aire de stockage des argiles à silex traitée à la chaux à la fin des travaux - vue du stock.

A la fin des terrassements en grande masse et après la réception de l'arase de terrassement, une planche de convenance a été réalisée en mai 2006 afin de valider le processus d'élaboration du matériau pour la couche de forme. Celui-ci, bien que décrit dans le CCTP, devait être validé avant le stade opérationnel par l'analyse du PAQ de l'entreprise, la discussion de sa procédure de travail et la réalisation d'une planche de convenance. Le dispositif de reprise et d'élaboration du matériau (Razel, Fig. 9) réellement mis en place par l'entreprise était fondé sur :

- une reprise du stock à la pelle ;

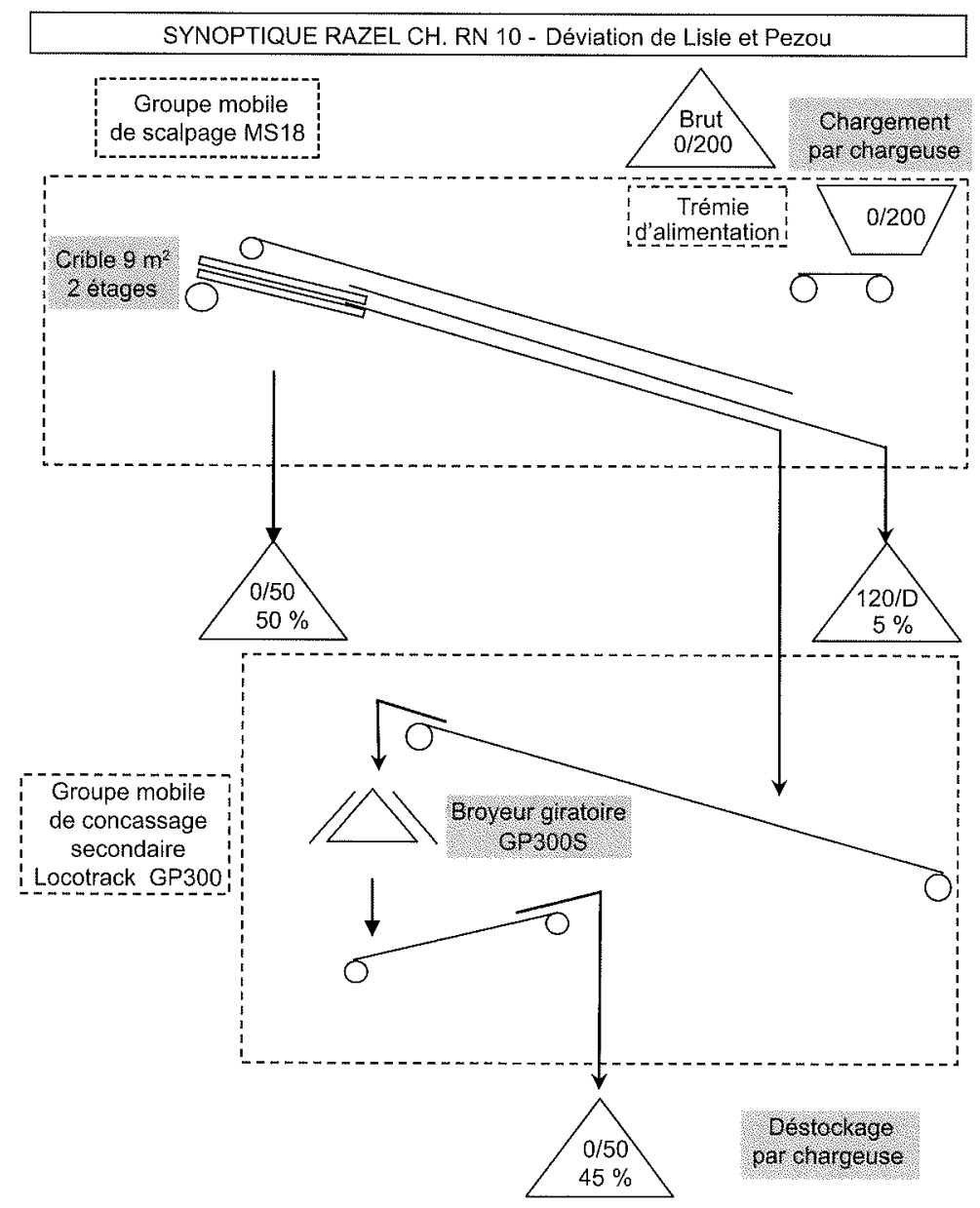




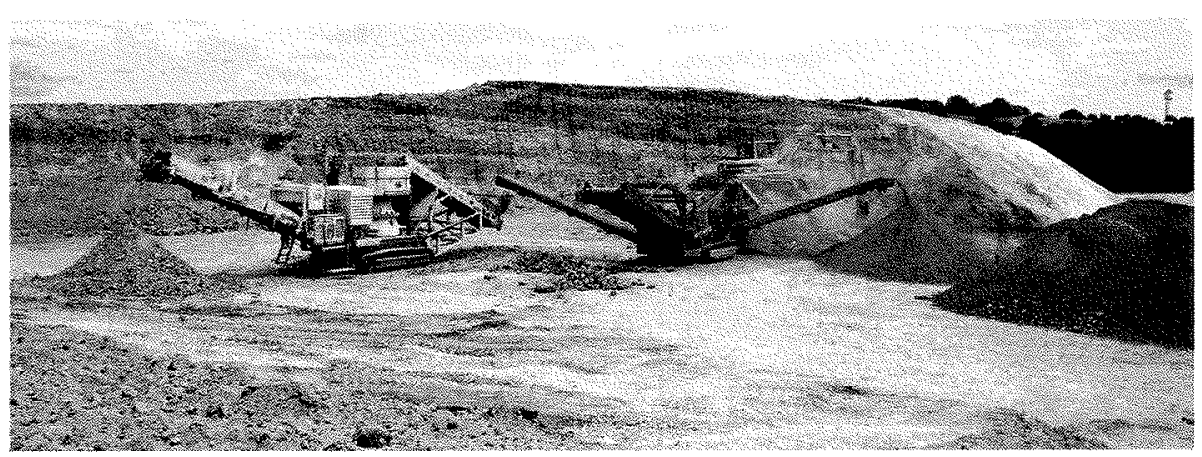

ff6 10 L'installation en juin 2006, avec le crible (au milieu), le concasseur (à gauche) et le stock recomposé (produit de criblage + silex concassés, à droite de la photo).

- un criblage pour éliminer les gros blocs de taille supérieure à $100 \mathrm{~mm}$ (Fig. 10) ;

- un concassage des éléments siliceux de taille comprise entre 70 et $100 \mathrm{~mm}$ dans un concasseur giratoire Nordberg LT 300 GP (Fig. 11) ;

- l'incorporation du matériau concassé au produit de criblage inférieur à $70 \mathrm{~mm}$.

Le retour d'expérience au moment des premières phases d'élaboration a permis de constater plusieurs points intéressants :

- les gros silex $\left(D_{\max }=250\right.$ à $\left.300 \mathrm{~mm}\right)$ visibles lors des reconnaissances géotechniques ou au moment du traitement à la charrue ne se retrouvaient plus au moment de la reprise à la pelle du stock, ni sur le crible supérieur de l'installation: de toute évidence le compacteur SP2 avait cassé ces blocs au cours de la montée du stock en couches minces ;

- le crible supérieur prévu pour éliminer les gros blocs de silex ne fonctionnait en réalité que pour éliminer (en grande majorité) les grosses mottes d'argile qui subsistaient dans le matériau après le traitement à la charrue. Le volume de « déchets 》 était sur ce poste très faible ;

- d'après les analyses géotechniques et les courbes granulométriques, le concasseur devait permettre la réduction des blocs de silex dont la taille était inférieure à $100 \mathrm{~mm}$. Il s'est avéré que ce poste était largement surdimensionné par rapport à l'importance du criblage : le volume de silex correspondait globalement à moins de $20 \%$ du volume repris par la pelle ;

- le traitement à la chaux vive a permis un désenrobage efficace des silex de leur gangue argileuse et a amélioré grandement l'opération de criblage.

Au final, après criblage, concassage et recomposition, le matériau obtenu correspondait à une grave sabloargileuse de type $0 / 63 \mathrm{~mm}$, classée $C_{1} B_{5}$ à $C_{1} B_{6}$ au sens de la norme NF P11-300 (Fig. 12), avec les caractéristiques suivantes : passant à $80 \mu \mathrm{m}$ compris entre 23,2 et $33,7 \%$; valeur au bleu des sols (selon NF P 94-068) comprise entre 1,32 et $1,75 \mathrm{~g}$ bleu $/ 100 \mathrm{~g}$ de sol.

Toutes les analyses granulométriques réalisées dans le cadre du suivi du PAQ ont montré que le matériau était homogène après la phase d'élaboration. L'exemple de la figure 13 illustre l'évolution du matériau notamment au niveau des fines. Cette évolution très intéressante est le résultat principalement de l'action de la chaux vive sur les particules argileuses et, dans une autre mesure, de l'incorporation d'une fraction de silex concassé corrigeant la courbe. La plasticité est devenue non mesurable permettant de passer d'une argile classée $\mathrm{A}_{3}$ à un $\mathrm{B}_{5}$.

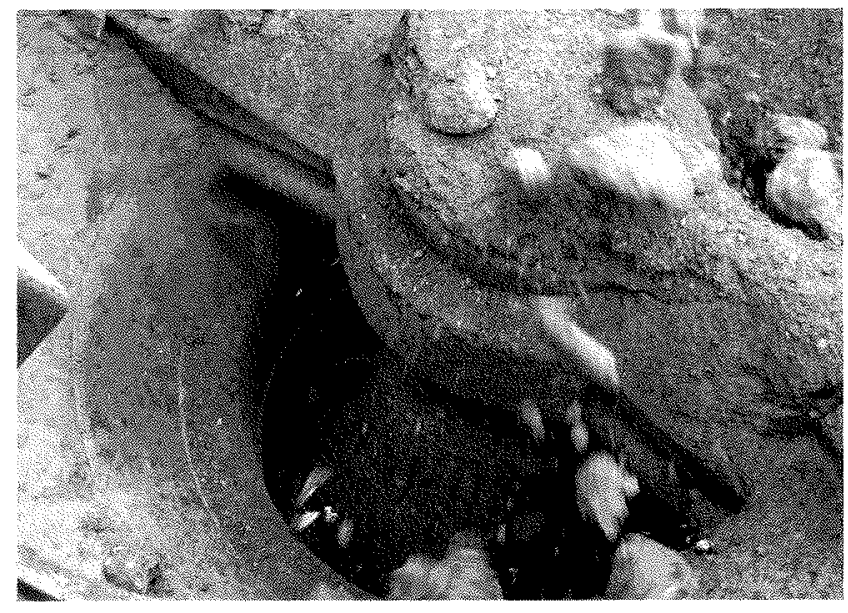

116 11 Concasseur giratoire en action, réduisant les silex de taille comprise entre 70 et $100 \mathrm{~mm}$.

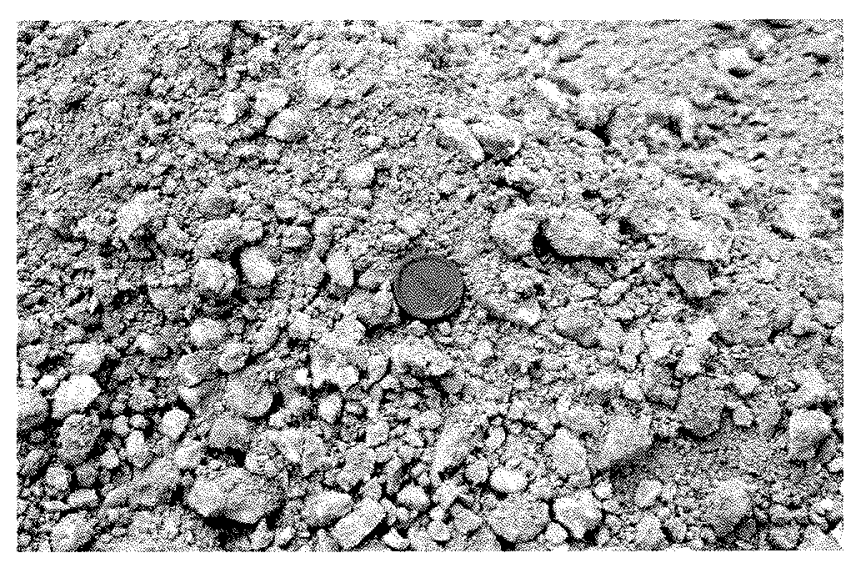

16. 12 Aspect de (c l'argile à silex » pré-traité à la chaux, criblé, concassé puis recomposé.

\section{2}

\section{Mise en œuvre}

Une fois élaboré, le matériau a été utilisé pour réaliser la couche de forme sur la section courante pour le traitement au liant hydraulique (Fig. 14 a et b). L'entreprise ayant réalisé sa propre étude de formulation, c'est un traitement à $5 \%$ de Ligex 111M10 qui a été retenu au lieu du dosage à $6 \%$ de ciment prévu au marché

La reprise et le criblage/concassage des matériaux ont été facilités par leur état hydrique relativement sec au coeur du stock. Cet avantage est vite devenu une 


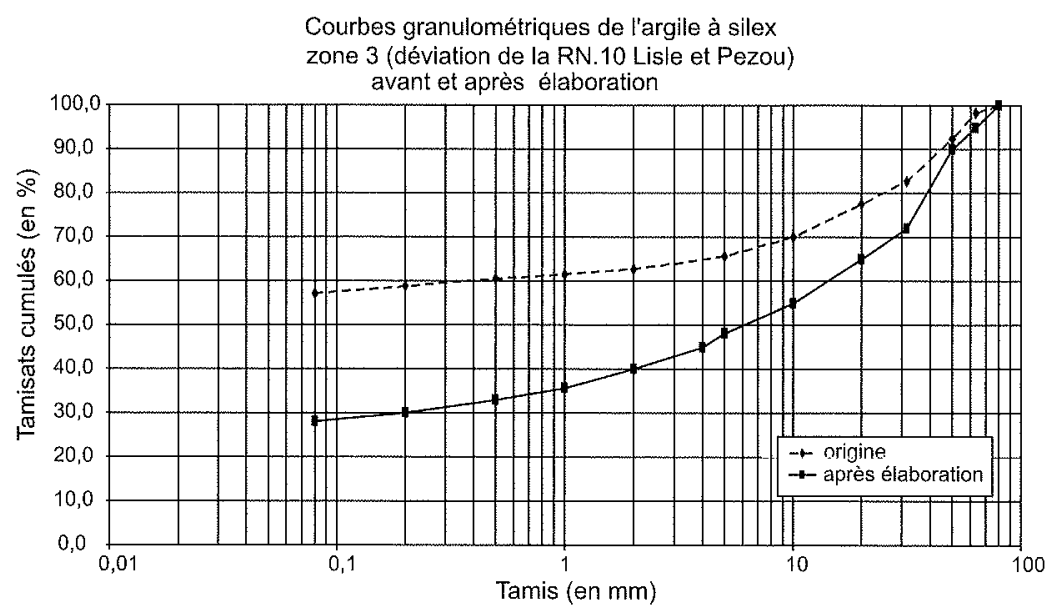

It6. 13 Courbes granulométriques de deux échantillons : l'un constitué avec le matériau d'origine, et l'autre après la phase d'élaboration.
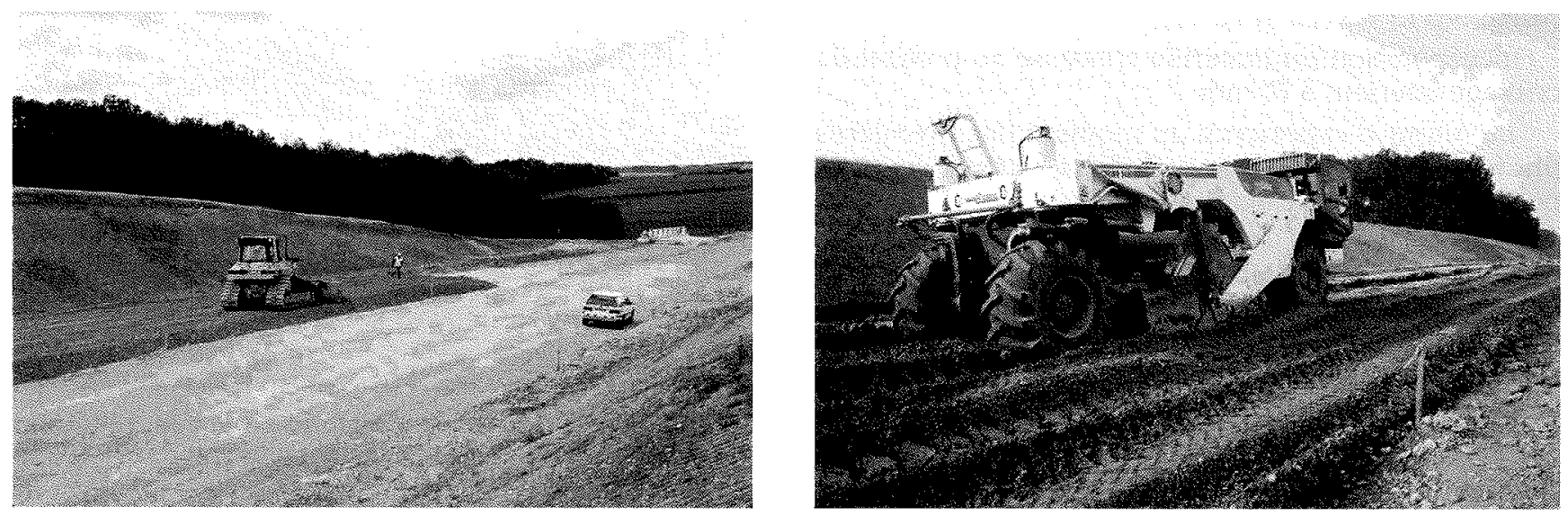

116. 14

a) Régalage du matériau en couche de forme, b) Malaxage en place au pulvimixeur (Wirtgen).

contrainte lorsqu'il a fallu ramener les états hydriques des matériaux à l'optimum Proctor avant leur traitement au liant hydraulique. Il fallait passer de teneurs en eau de départ comprises entre 14,6 et $21,7 \%$ à un optimum $\mathrm{w}_{\text {opn }}$ de $23 \%$. Le changement d'état hydrique a donc été difficile à gérer, dans un contexte météorologique très évaporant et durant un été où les ressources en eau n'étaient pas faciles à mobiliser (été 2006). L'incorporation d'eau par des arroseuses queue de carpe était réalisée la veille du traitement, et l'homogénéisation effectuée par une passe de pulvimixeur.

Après épandage du liant et malaxage par deux passes de pulvimixeur, le compactage a été assuré par des compacteurs à cylindre vibrant classés V5 en appliquant les consignes du Guide pour la réalisation des remblais et des couches de formes (LCPC-SETRA, 1992). Le réglage fin était assuré par des niveleuses, et la fermeture de surface par un passage de cylindre lisse. Un enduit de cure gravillonné réalisé à l'avancement a permis de maintenir les états hydriques et de protéger la couche de forme jusqu'à la réalisation des couches de chaussée par une autre entreprise.

\section{6}

\section{Réception et épreuves d'information}

La couche de forme a été réceptionnée en déflexion par l'entreprise (mesures à la poutre) et par le LRPC de
Blois (au déflectographe Lacroix). La valeur maximale fixée au CCTP était de 80/100 mm. Sur un découpage en zones homogènes, la très grande majorité des sections a obtenu des valeurs moyennes de déflexion comprises entre 10 et 25/100 de mm (Fig. 15) ce qui a permis de prononcer la réception de la couche de forme en PF2. Les anomalies locales ont fait l'objet d'investigations complémentaires

Les carottages prévus dans le cadre d'une opération de recherche du LCPC ont permis de pousser un peu plus loin les investigations sur la couche de forme. La campagne prévoyait la réalisation de 41 carottes réalisées au carottier double enveloppe en diamètre $110 \mathrm{~mm}$ extérieur, réparties uniformément sur la surface de la couche de forme depuis le profil 76 jusqu'au profil 302. Chaque carotte a fait l'objet d'un compte rendu détaillé permettant de faire un travail de synthèse sur (Fig. 16) : le contrôle des épaisseurs, la vérification des modules et des résistances en compression diamétrale, la qualité visuelle du matériau traité, la densité humide mesurée au banc gamma.

Les résultats obtenus ont été instructifs pour connaître les épaisseurs réellement mises en œuvre. Les performances mécaniques ont été plus difficiles à évaluer, car le mode de récupération par carottage des matériaux a été très perturbé par la présence des silex. 


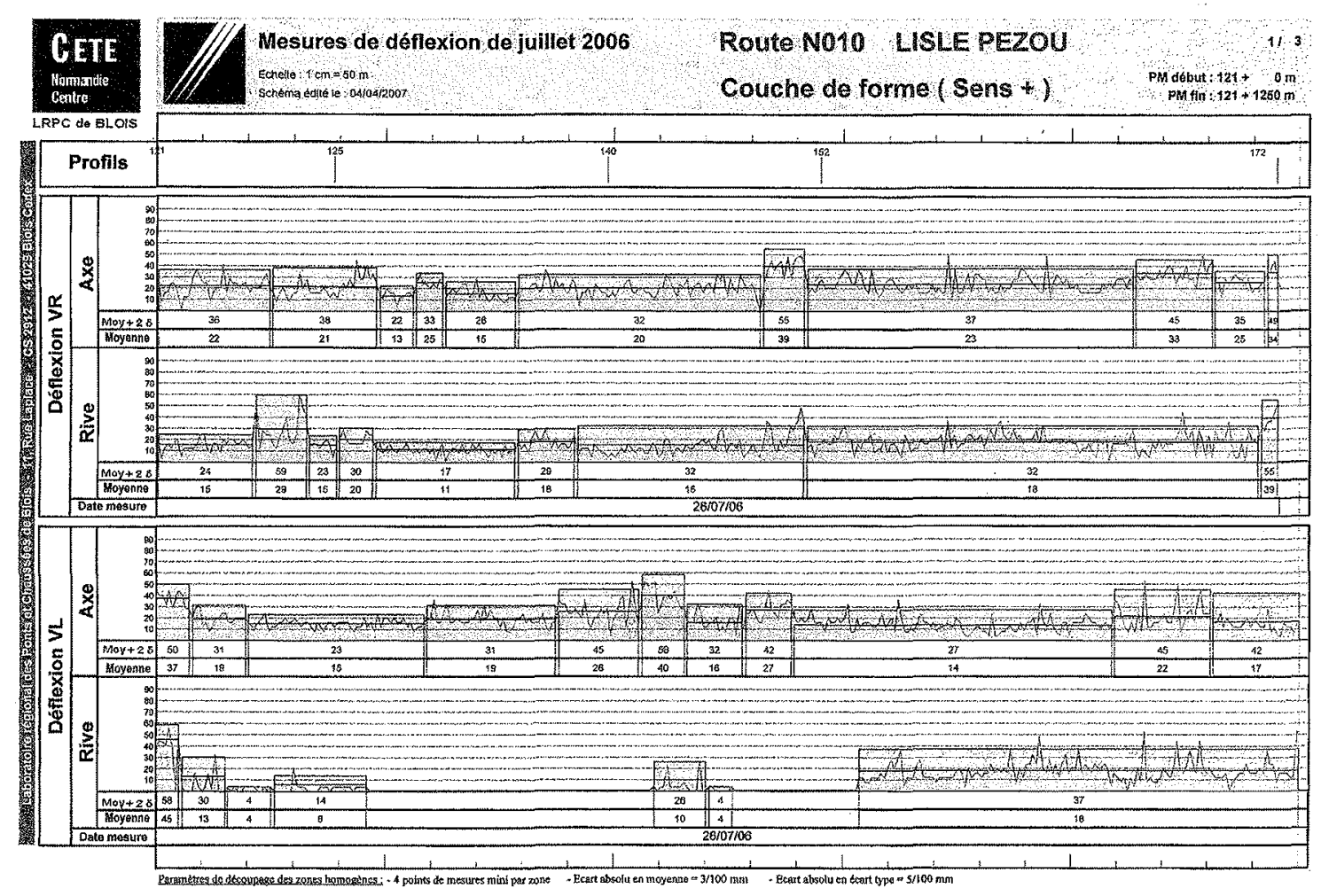

Hc. 1 Mesures de déflexion sur un section de la RN 10 au déflectographe Lacroix.

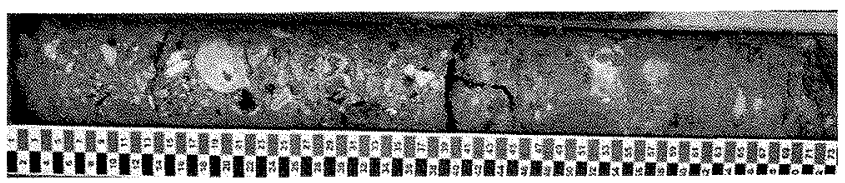

2. 16.16 Exemple de carottage de la couche de forme (échelle en $\mathrm{cm}$ ).

\section{5}

\section{Conclusion}

L'expérimentation a permis de montrer la faisabilité du réemploi des argiles à silex même très plastiques, et de leur valorisation en couche de forme avec des processus simples. Cette expérience doit cependant être encadrée pour pouvoir s'appliquer avec succès sur d'autres chantiers :
- la réussite repose sur l'identification d'un gisement apte à fournir un matériau homogène pour les besoins de la couche de forme ;

- l'entreprise doit maîtriser le processus depuis le traitement à la chaux vive, le stockage, le concassage jusqu'à la mise en cuvre définitive et le traitement au ciment, avec un suivi qualité approprié ;

- le traitement sera réussi moyennant l'incorporation du juste dosage en chaux et en ciment, conforme à l'étude. De ce point de vue une analyse plus fine des incertitudes ou de la variabilité de certains paramètres (argilosité, dosage en liant) permettra d'obtenir de meilleurs résultats (PF3).

Ce retour d'expérience ouvre de nouvelles perspectives dans un contexte globalement en pénurie de matériaux nobles, dans une période où la valorisation des excédents de chantier est fortement incitée, et ceci grâce à l'essor des techniques de traitement et à la disponibilité de matériels mobiles performants.

\section{Bibliographie}

AFNOR - NFP 11-300 Exécution des terrassements. Classification des matériaux utilisables dans la construction des remblais et des couches de forme d'infrastructures routières, 1992

LCPC-SETRA - Traitement des sols à la chaux et/ou aux liants hydrauliques. Guide technique, 2000, $240 \mathrm{p}$.

LCPC-SETRA - Guide pour la réalisation des remblais et des couches de formes. Guide technique vol. 1 et 2, 1992.

Laignel B. - Utilisation des formations résiduelles à silex comme matériaux de substitution ou de complément des granulats alluvionnaires. Rapport provisoire de thèse, université de Rouen, 1996.

Ledee V. - Étude bibliographique sur l'exploitation des formations géologiques a argile et éléments de silex de l'ouest et du sud du Bassin parisien. Rapport de recherche LCPC, 1998.

Spencer C. Laignel B. Le Berre P. - Les argiles à silex de l'ouest du Bassin parisien. Un matériau de substitution possible aux granulats alluvionnaires. Revue Mines et Carrières, mars 2000, p. 34-40. 